\title{
The ion-step-induced response of membrane-coated ISFETs: theoretical description and experimental verification*
}

\author{
R. B. M. Schasfoort \\ TNO Institutes, PO Box 360, 3700 AJ Zeist, The Netherlands \\ P. Bergveld, R. P. H. Kooyman \& J. Greve \\ University of Twente, PO Box 217, 7500 AE Enschede, The Netherlands
}

(Received 4 May 1990; revised version received 20 October 1990; accepted 15 November 1990)

\begin{abstract}
Recently a new method was introduced to operate an immunological field effect transistor (ImmunoFET). By changing the electrolyte concentration of the sample solution stepwise (the so-called ion-step), a transient diffusion of ions through the membrane-protein layer occurs, resulting in a transient membrane potential, which is measured by the ImmunoFET. It became apparent that the maximum of the membrane potential is a function of $\mathrm{pH}$, owing to a pH-dependent charge density caused by the amphoteric nature of the embedded proteins in the membrane of the ImmunoFET. At a certain $\mathrm{pH}$ value, which is called the inversion point $\left(\mathrm{pI}^{\prime}\right)$, the membrane potential changes sign. This inversion point is characteristic of the type of protein and the type of membrane and depends on the isoelectric point, the titration curve and the concentration of all amphoteric groups in the membrane.

In this paper an attempt is made to establish a theoretical basis for the ionstep method. Because there is no model which describes ion transport in charged membranes and its dynamic behaviour as a result of the ion-step, an existing equilibrium theory has been adapted. The well-known Teorell-MeyerSievers (TMS) theory, which describes the membrane potential for charged membranes, is used as a framework. The adapted TMS model was verified by experimental data.
\end{abstract}

Keywords: biosensors, ion-sensitive field effect transistor (ISFET), immunosensor, immunoFET, ion-step, isoelectric point, protein membrane, charged membrane. lysozyme. TMS theory. membrane potential. Donnan potential. diffusion potential.

\footnotetext{
* Paper presented at Biosensors '90, Singapore, 2-4 May 1990.
} 


\section{INTRODUCTION}

More than a decade ago researchers started to investigate the possibility of direct detection of an immunological reaction by means of electrodes and/or modified ISFETs. A review of the published efforts to construct an immunosensor was given by Aizawa (1987). All of these immunosensors are of the static type, which means that as a result of the antibody-antigen immune reaction, a shift in the d.c. potential of a certain (modified) electrode occurs. Often these immunoelectrodes suffer from drift, insensitivity, nonreproducibility etc. (Collins \& Janata, 1982).

Recently a new 'dynamic' method was introduced by Schasfoort et al. (1990) for monitoring an immunological reaction occurring in a porous membrane covering an ISFET. This method is based on the measurement of transient membrane potentials $\Delta \phi_{\mathrm{m}}$, induced by a sudden change in the electrolyte concentration. $\Delta \phi_{m}$ can be modulated by applying ion-steps to the system with $\mathrm{pH}$ as a parameter. At a certain $\mathrm{pH}$ value, which is called the inversion point $\mathrm{pI}^{\prime}, \Delta \phi_{\mathrm{m}}$ changes sign. This inversion point is characteristic for the type of protein (i) in the membrane (reflected by the isoelectric point and the titration curve of protein $i$ ) and the concentration of each protein $i$ in the membrane. An empirical formula was suggested in order to quantitatively account for the observed effects:

$\Delta \phi_{\mathrm{m}}=\left[\sum_{i=0}^{i=n} S_{i} c_{i}(\mathrm{pH}-\mathrm{pI})\right] \frac{R T}{F} \ln \left(\frac{a_{2}}{a_{1}}\right)$

where $\Delta \phi_{\mathrm{m}}$ is the extremum of the membrane potential after the ion-step, $\mathrm{pI}_{i}$ is the isoelectric point of protein $i, c_{i}$ is the average concentration of protein $i$ in the membrane, $S_{i}$ is a sensitivity factor, which is a measure of $\delta Q_{i} / \delta \mathrm{pH}\left(Q_{i}\right.$ represents the charge of protein $i$ ) and depends on the titration curve of the protein $i$, and is characteristic for each particular protein $(i=0$ denotes a bare membrane). $S_{i}$ has the dimensions of reciprocal concentration (litre $\mathrm{mol}^{-1}$ ), $R, T$ and $F$ have their usual meanings, and $a_{1}$ and $a_{2}$ are the $\mathrm{KCl}$ activities before and after the ion-step.

The notion that a $\mathrm{pH}$-dependent difference exists between cation and anion mobility in the protein membrane will constitute the central theme of the considerations in this paper. Firstly, a short review of the TMS theory will be given: the separate contributions of the boundary and diffusion potentials to the total membrane potential are illustrated. Secondly, the TMS theory, which was derived for the steady-state model, will be applied to describe the dynamic potential response as a result of the ion-step. Finally, experimental data are given in order to verify both the adapted TMS model and the empirical formula as mentioned above. Some possible refinements and suggestions for further theoretical description and experimental verification are indicated.

\section{THEORETICAL DESCRIPTION OF THE MEMBRANE POTENTIAL TRANSIENT}

\section{Diffusion of ions through a charged membrane}

Consider a two-compartment system with different electrolyte concentrations $\left(c_{\mathrm{s} 1}\right.$ and $c_{\mathrm{s} 2}$ ) separated by a membrane with fixed charge density $c_{\mathrm{x}}$. The diffusion of ions through the membrane is influenced by the fixed charges. The different electric mobilities $\left(u_{j}=(k T / z e) D_{j}\right)$ of co- and counter-ions (ions with respectively the same and opposite charges as the fixed membrane charges) in the membrane results in a contribution to the membrane potential.

The original theory of diffusion through charged membranes with respect to the electric potential generation was given by Teorell (1935) and Meyer and Sievers (1936) (TMS theory).

An example of recent publications with respect to TMS theory is given by Kaibara et al. (1986) and Higuchi and Nakagawa (1987) who studied ion transport and membrane potential generation in charged membranes. An evaluation of several fixed charge theories for membrane potential is given by Beg et al. (1977).

In the TMS theory the following assumptions are made:

(a) The ion concentrations $c^{+}, c^{-}$in the membrane phase differ only in the $z$-direction perpendicular to the membrane, implying that no concentration gradient exists parallel to the membrane surfaces.

(b) The fixed charges are homogeneously distributed over the membrane phase which is considered to be of uniform thickness $\delta$.

(c) The water transport is negligible and no 
swelling of the membrane is taken into account.

(d) During the diffusion process, the diffusion coefficients for $\mathrm{c}^{+}$and $\mathrm{c}^{-}$are independent of their positions in the membrane.

(e) Activity coefficients are assumed to be unity. Thus activities can be replaced by concentrations (diluted solutions).

(f) Electroneutrality is considered to exist in the system.

\section{The Donnan or boundary potential}

As a consequence of the electroneutrality condition within the membrane, the counter-ion concentration within the membrane will always be larger than the co-ion concentration. The difference between co- and counter-ion concentration balances the effective fixed charge density $\left(c_{x}\right)$ of the membrane (thus in the membrane $c^{+}-c^{-}+c_{\mathrm{x}}=0$ for a $1-1$ salt; note that the charge density $c_{x}$ also bears the sign of the membrane charge, implying that $c_{x}$ can also have a negative value). Because at both sides of the membrane the electrolyte concentration is different, the ion distributions at both boundaries will be adapted. As a consequence the boundary potentials or Donnan potentials at the membrane electrolyte interfaces are also different. The Donnan potential at boundary 2 is represented by (Moore, 1976):

$$
\begin{aligned}
\Delta \phi_{\mathrm{don} 2} & =\frac{R T}{F} \ln \left(\frac{c_{\delta}}{c_{\mathrm{s} 2}}\right) \\
& =\frac{R T}{F} \ln \left[\frac{\left(4 c_{\mathrm{s} 2}{ }^{2}+c_{\mathrm{x}}^{2}\right)^{1 / 2}+c_{\mathrm{x}}}{2 c_{\mathrm{s} 2}}\right]
\end{aligned}
$$

$\Delta \phi_{\text {don2 }}$ is the Donnan potential between the membrane and solution 2 (see Fig. 1). Note that if $c_{\mathrm{x}}$ has the opposite sign, the sign of $\Delta \phi_{\mathrm{don} 2}$ is also reversed. A similar equation holds for $\Delta \phi_{\text {don } 1}$.

(Note: in hydrophobic membranes the standard chemical potential in the membrane phase is different from that in the bulk. A salt partition $\left(K_{\mathrm{s}}\right)$ between the (bulk) membrane phase and the (bulk) aqueous phase will exist. However, if hydrophilic charged membranes are used, only the partition coefficients of the co- and counter-ions $\left(K^{+}\right.$and $\left.K^{-}\right)$in the porous membrane, which depend on the Donnan potential differ. The salt partition coefficient can be approximated as $K_{\mathrm{s}}=\left(K^{+} K^{-}\right)^{1 / 2}$ (Push, 1986), and is here assumed to be unity.)

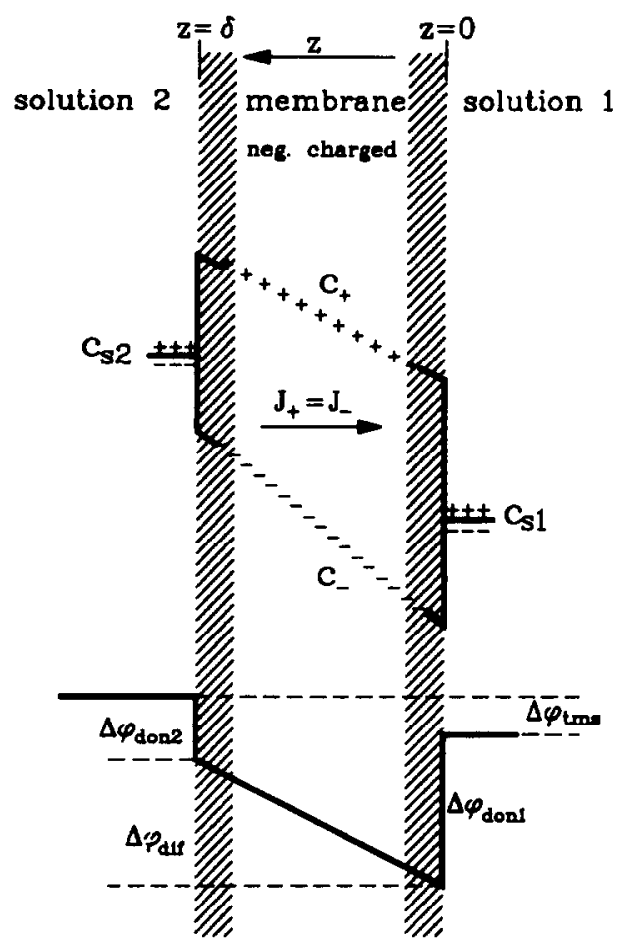

Fig. 1. Schematic representation of a membrane which separates two solutions having electrolyte concentrations $c_{\mathrm{s} 2}$ and $\mathrm{c}_{\mathrm{s}}$. The concentration distribution of cations $\mathrm{c}^{+}(+)$ and anions $\mathrm{c}^{-}(-)$is given for the case of fixed negative charges present in the membrane. The membrane potential $\Delta \phi_{\text {tms }}$ is created by the boundary or Donnan potentials $\Delta \phi_{\text {don: }}$ and $\Delta \phi_{\text {donl }}$ and the diffusion potential $\Delta \phi_{d t r}$. Note that the difference in the Donnan potentials $\Delta \phi_{\text {don } 2}-\Delta \phi_{\text {donl }}$ counteracts the diffusion potential $\Delta \phi_{\text {dif }}$.

\section{The diffusion potential}

Ions diffuse in the absence of an external electric field, if there is a difference in the chemical potential between different parts of the system. If there are steady fluxes of anions and cations through the membrane, the system is not in a state of equilibrium but rather in a steady state, characterised by the fact that the total electric current through the membrane is zero.

As a consequence of the electroneutrality condition, the counter-ion concentration within the membrane is always larger than the co-ion concentration. Moreover, if there is no net electric transport, the flux of the cations equals the flux of the anions $\left(J^{+}=J^{-}\right)$. This implies that the diffusion coefficient of the co-ions is increased with respect to the diffusion coefficient of the counter-ions.

By integrating the Nernst-Planck equations (MacGillivray, 1968; Aquilella et al., 1987) 
Teorell, Meyer and Sievers derived the following relationship for the diffusion potential $\Delta \phi_{\text {dif }}$ for a 1-1 salt system.

$$
\Delta \phi_{\mathrm{dif}}=U \frac{R T}{F} \ln \left[\frac{\left(4 c_{\mathrm{s} 2}{ }^{2}+c_{\mathrm{x}}{ }^{2}\right)^{1 / 2}-c_{\mathrm{x}} U}{\left(4 c_{\mathrm{s} 1}{ }^{2}+c_{\mathrm{x}}\right)^{1 / 2}-c_{\mathrm{x}} U}\right]
$$

where the value of $U$ follows from diffusion coefficients $\left(D^{+}\right.$and $\left.D^{-}\right)$of cations and anions in the membrane: $U=\left(D^{+}-D^{-}\right) /\left(D^{+}+D^{-}\right)$. The membrane potential $\left(\Delta \phi_{\mathrm{tms}}\right)$ is the sum of the Donnan potentials at the boundaries of the membrane and the diffusion potential (see Fig. 1):

$$
\Delta \phi_{\mathrm{tms}}=\left(\Delta \phi_{\mathrm{don} 2}-\Delta \phi_{\mathrm{don} 1}\right)+\Delta \phi_{\mathrm{dif}}
$$

Thus:

$$
\begin{aligned}
\Delta \phi_{\mathrm{tms}}= & \frac{R T}{F}\left\{\ln \left[\frac{c_{\mathrm{s} 1}}{c_{\mathrm{s} 2}} \frac{\left(4 c_{\mathrm{s} 2}{ }^{2}+c_{\mathrm{x}}{ }^{2}\right)^{1 / 2}+c_{\mathrm{x}}}{\left(4 c_{\mathrm{s} 1}{ }^{2}+c_{\mathrm{x}}{ }^{2}\right)^{1 / 2}+c_{\mathrm{x}}}\right]\right. \\
& \left.+U \ln \left[\frac{\left(4 c_{\mathrm{s} 2}{ }^{2}+c_{\mathrm{x}}{ }^{2}\right)^{1 / 2}-c_{\mathrm{x}} U}{\left(4 c_{\mathrm{s} 1}{ }^{2}+c_{\mathrm{x}}{ }^{2}\right)^{1 / 2}-c_{\mathrm{x}} U}\right]\right\}
\end{aligned}
$$

This is the equation originally given by Teorell, Meyer and Sievers (Teorell, 1935; Meyer \& Sievers, 1936). Note that for $c_{\mathrm{x}}=0$ the well-known Nernst-Planck equation is obtained: $\Delta \phi_{\mathrm{tms}}$ $=U R T / F \ln \left(c_{\mathrm{s} 2} / c_{\mathrm{s} 1}\right)$. The membrane potential then has a value of $59 \mathrm{U} \mathrm{mV}_{\text {decade }}{ }^{-1}$.

\section{Influence of the effective charge density on the value of $\boldsymbol{U}$}

The membrane potential $\Delta \phi_{\mathrm{tms}}$ can be calculated by using eqn (5), if the values of both $U$ and $c_{\mathrm{x}}$ are known. However, these values are not independent of each other. Therefore a relationship is needed which correlates these parameters. In the work of Demisch and Push $(1979,1980)$ a starting point for the derivation of this relationship is given, as will be reviewed here (Kimura et al., 1984).

In Fig. 2 the membrane potential $\Delta \phi_{\text {tms }}$, the difference in the Donnan potentials $\Delta \phi_{\text {don2 }}$ $\Delta \phi_{\text {don1 }}$ and the diffusion potential $\Delta \phi_{\text {dif }}$ are plotted as functions of $c_{\mathrm{s} 2}$ for fixed values of $c_{\mathrm{s} 1}, U$ and $c_{\mathrm{x}}$.

It is shown that the membrane potential curve consists of two branches with opposite slopes. If $c_{\mathrm{s} 2} \ll\left|c_{\mathrm{x}}\right|$, here denoted as the Donnan region, the difference in the Donnan potentials has a marked influence on the membrane potential,

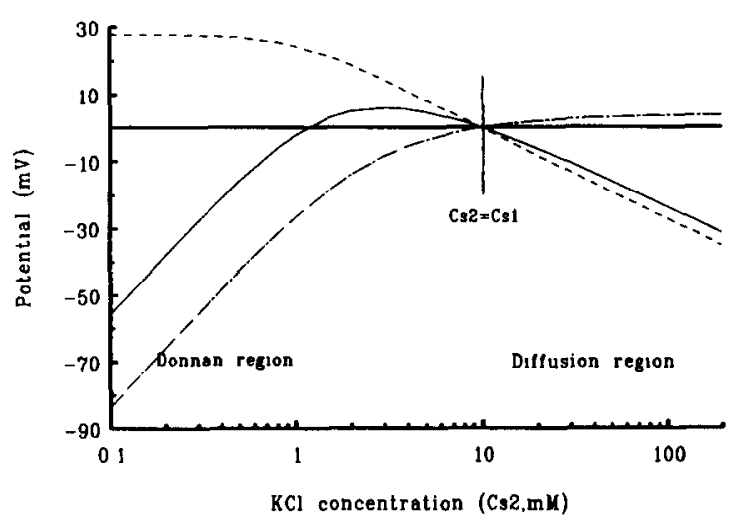

Fig. 2. Membrane potential $\Delta \phi_{\text {tms }}$ (-), the difference in the Donnan potentials $\Delta \phi_{\text {don } 2}-\Delta \phi_{\text {don I }}(-.-$ ), and the diffusion potential $\Delta \phi_{d i j}(---)$ plotted as a function of the salt concentration $c_{s 2}$ for a fixed value of $\mathrm{c}_{\mathrm{s} l}=10 \mathrm{~mm}, \mathrm{U}=0.45$ and $\mathrm{c}_{x}=3 \mathrm{~mm}$. Two regions can be distinguished for $\Delta \phi_{\text {tms }}$ as explained in the text.

resulting in a Nernstian behaviour (thus $59 \mathrm{mV}$ per decade). If $c_{\mathrm{s} 2} \gg\left|c_{\mathrm{x}}\right|$, i.e. the diffusion region, the diffusion potential contributes most to the membrane potential. In this region, the slope of the membrane potential as a function of $c_{\mathrm{s} 2}$ for a fixed $c_{s 1}$ is determined by the value of $U$. In general, the difference in the Donnan potentials counteracts the diffusion potential. This counteracting effect also results in the fact that a $c_{\mathrm{s} 2}$ can be found (denoted as $c_{\mathrm{s} 2 \text {. ex }}$ ) where the membrane potential $\Delta \phi_{\mathrm{tms}}\left(c_{\mathrm{s} 2}\right)$ exhibits an extremum. This extremum reflects the intrinsic parameters of the membrane but also depends on intrinsic mobility differences of the co- and counter-ions. By differentiating the TMS equation with respect to $c_{\mathrm{s} 2}$, the following equation is obtained:

$$
U= \pm\left[c_{\mathrm{x}}^{2} /\left(4 c_{\mathrm{s} 2, \mathrm{ex}}{ }^{2}+c_{\mathrm{x}}{ }^{2}\right)\right]^{1 / 2}
$$

The sign of $U$ is the same as the sign of $c_{\mathrm{x}}$.

In Fig. 3 the membrane potential, the result of the Donnan potentials and the diffusion potential are shown as a function of $c_{\mathrm{x}}$ at constant electrolyte concentrations $\left(c_{\mathrm{s} 2}=40 \mathrm{mM}, c_{\mathrm{s} 1}=10\right.$ $\mathrm{mm}$ ) and for $c_{\mathrm{s} 2 \text {, ex }}=3 \mathrm{~mm}$.

Equation (6) was used to calculate $U$. The justification for this procedure stems from the experimental results of Demisch and Push (1979), who showed that $c_{\mathrm{s} 2 \text {. ex }}$ remains constant for different membrane charge densities. However, this assumption is only true for a special case as will be described in the Results and Discussion section.

It can be observed from Fig. 3 that in this case, 


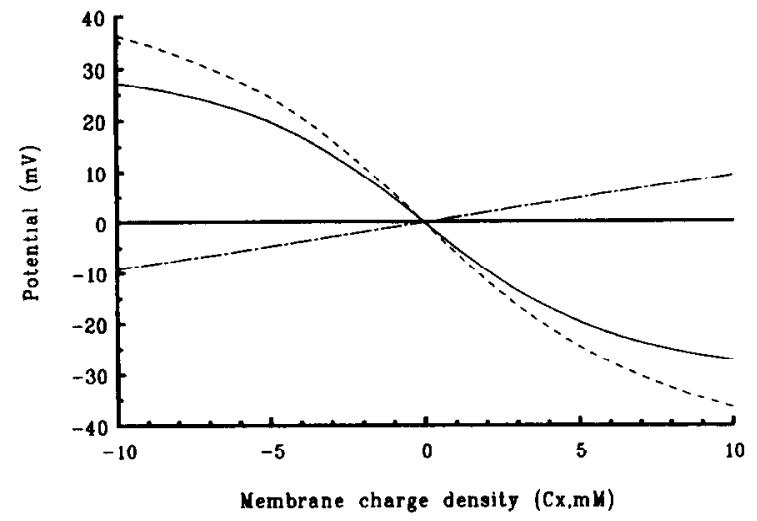

Fig. 3. Membrane potential $\Delta \phi_{\text {rms }}(\longrightarrow)$, the difference in the Donnan potentials $\Delta \phi_{d o n} 2-\Delta \phi_{d o n 1}(-,-)$, and the diffusion potential $\Delta \phi_{\text {dif }}(---)$ plotted as a function of the membrane charge density $\mathrm{c}_{x}$ for fixed salt concentrations $\mathrm{c}_{s 1}=10 \mathrm{~mm}$ and $\mathrm{c}_{s_{2}}=40 \mathrm{mM}$. the extremum is at $\mathrm{c}_{\mathrm{s}_{2} \text {, ex }}=3 \mathrm{~mm}$ (see Fig. 2) and $\mathrm{U}_{0}=0$.

which is close to our experimental situation, the diffusion potential contributes most to the net membrane potential. If the membrane charge density is zero, the membrane potential is zero. This is only valid if the value of $U$ in an uncharged membrane $\left(U_{0}\right)$ is very small (or zero) (e.g. for $\mathrm{KCl}, U_{0}=0.018$ ). If a salt with different diffusion coefficients for cations and anions in an uncharged membrane is used, then a diffusion potential is present at $c_{\mathrm{x}}=0$.

\section{The ion-step}

Before proceeding, some comment is necessary on the extent to which the above reviewed theory is valid for the dynamic ion-step method applied to a solid-state supported membrane system.

During the ion-step the salt concentration at the solution/membrane interface $\left(c_{\mathrm{s} 2}\right)$ undergoes a sudden change while, depending on the thickness of the membrane, the concentration at the membrane/substrate contact $\left(c_{\mathrm{s} 1}\right)$ remains unchanged for a certain period of time.

In order to calculate the membrane potential at time $t, \Delta \phi_{\mathrm{tms}}(t)$, by using the TMS equation, it is important to know how fast the ion concentrations change as a result of diffusion through the membrane. The change in concentration as a result of diffusion is illustrated in Fig. 4 (in fact the salt diffusion in an uncharged membrane is considered here). Time-dependent diffusion equations are necessary, which should be inserted in the TMS equation.

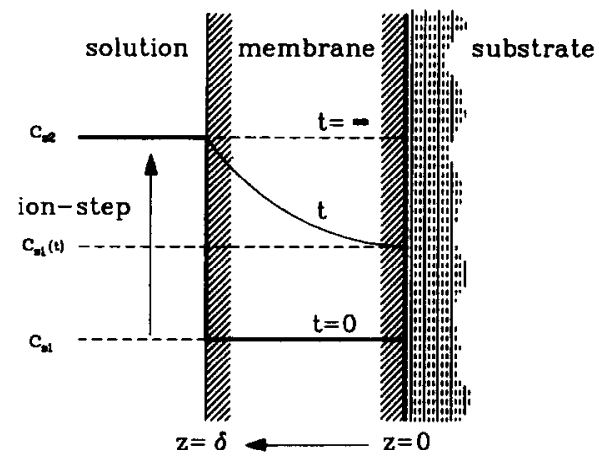

Fig. 4. Diffusion model for a membrane deposited on a substrate and exposed to an ion-step from salt concentration $\mathrm{c}_{s l}$ to $\mathrm{c}_{s 2}$. The concentration $\mathrm{c}_{s l(t)}$ at the substrate/ membrane interface depends on the membrane thickness $\delta$ and the effective diffusion coefficients in the membrane.

The ion diffusion can be described by Fick's second law of diffusion considering the given boundary conditions. Thus the following equation has to be solved:

$$
\frac{\partial c(z, t)}{\partial t}=D_{\mathrm{s}} \frac{\partial^{2} c(z, t)}{\partial z^{2}}
$$

$D_{\mathrm{s}}$ then reflects the salt (mean) diffusion coefficient and is independent of $z$ for both positive and negative ions. Nernst showed that for electrolytes of the 1-1 type the appropriate average is (Moore, 1976):

$$
D_{\mathrm{s}}=\frac{2 D+D^{-}}{D^{+}+D^{-}}
$$

The concentration profile can be determined at certain time intervals by numerically solving eqn (7). The membrane potential then can be calculated from the different quasi steady-state situations at different positions of $z$ in the membrane. However, a much simpler approximate analytical expression for the concentration at the membrane/substrate interface at different time intervals is given by Morf et al. (1975):

$c_{\mathrm{s} 1(t)}=c_{\mathrm{s} 1(0)}+\left(c_{\mathrm{s} 2}-c_{\mathrm{s} 1(0)}\right)[1-\exp (-t / \tau)]$

with the time constant

$$
\tau=\frac{4 \delta^{2}}{\pi^{2} D_{s}}
$$

$c_{\mathrm{s} 1(t)}$ is the concentration at the solid-state surface at each time $t>0, c_{\mathrm{s} 1(0)}$ and $c_{\mathrm{s} 2}$ are the concentrations in the bulk solution at the time $t<0$ and $t>0$ respectively and $\delta$ is thickness of the membrane. In the following it will be indicated to 
which extent this expression (9) holds in the ionstep experiments.

If a time-dependent concentration $c_{s 1(t)}$ and typical values for charge density and $U$ are inserted into eqn (5), a time-dependent membrane potential can be calculated. The concentration at $z=0$ (Fig. 4) as described by eqn (9), determines the membrane potential at time $t$. Also an experimental value for the duration of the ion-step $\left(t_{\text {step }}\right)$ must be included in the simulation (the time in which $c_{\mathrm{s} 2}$ reaches its new value; it was simulated as an exponential concentration change as a function of time, according to eqn (9), with time constant $t_{\text {step }}$ ). In Fig. 5 a graph of simulated transients for different membrane thicknesses is shown.

$D_{\mathrm{s}}$ is assumed to be $5 \times 10^{-12} \mathrm{~m}^{2} \mathrm{~s}^{-1}\left(D_{\mathrm{s}}\right.$ in a charged membrane (Demisch \& Push, 1979)), $c_{\mathrm{x}}=-7.20 \mathrm{mM}, U=-0.77, t_{\text {step }}$ is $0.05 \mathrm{~s}$. The charge density in the membrane and $t_{\text {step }}$ primarily determines the extremum of the membrane potential. However, the width and height of the transient are also determined by the membrane thickness. It can be seen from Fig. 5 that the extremum of the membrane potential of the $3 \mu \mathrm{m}$ membrane is substantially affected owing to the experimental time interval in which an ion-step is supposed to be accomplished. In our experiments, the membrane thickness is about $5 \mu \mathrm{m}$, and $t_{\text {step }} \approx 0.05 \mathrm{~s}$, which indicates that the extremum of the membrane potential is already affected by the non-ideality of the ionstep. However, the mean diffusion coefficient of

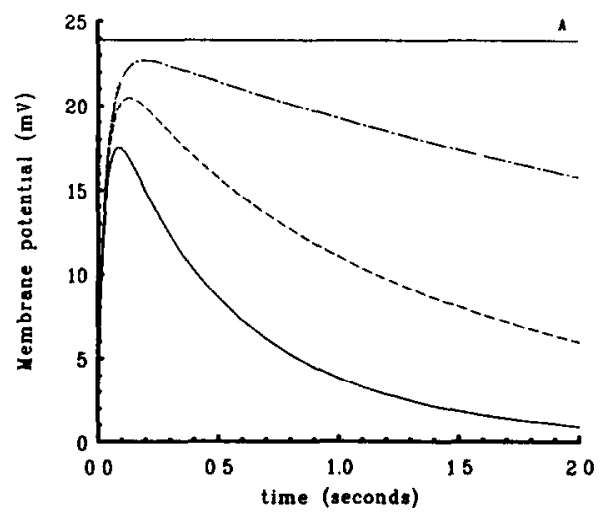

Fig. 5. Simulated transient $\left(\Delta \phi_{\text {tmstrt }}\right)$ after an ion-step from $\mathrm{c}_{\mathrm{s} I}(0)=10 \mathrm{~mm}$ to $\mathrm{c}_{\mathrm{s} 2}=40 \mathrm{~mm} \quad\left(\mathrm{t}_{\mathrm{ste})}=0.05 \mathrm{~s}\right)$. $\mathrm{D}_{\mathrm{s}}=5 \times 10^{-12} \mathrm{~m}^{2} \mathrm{~s}^{-1}, \mathrm{c}_{\mathrm{r}}=-7.2 \mathrm{~mm}$ and $\mathrm{U}=-0.77$ for four different thicknesses of the membrane: $\cdots, 20 \mu \mathrm{m}$; -. $10 \mu \mathrm{m}$ : ----. $5 \mu \mathrm{m}:-3 \mu \mathrm{m}$. The horizontal line $A$ represents the maximum theoretical membrane potential $\left(\mathrm{c}_{s I}=\right.$ constant and $\mathrm{t}_{\text {step }}=0$ ). the ions in the membrane determines to a large extent whether $t_{\text {step }}=0.05$ results in an ideal ionstep and it is probably too high an estimate. An indication that near-ideal ion-steps take place with our experimental system is that $\Delta \phi_{\mathrm{m}}$ values of more than $30 \mathrm{mV}$ in the standard procedure (with a theoretical maximum of $35 \mathrm{mV}$ ) are obtained at high effective charge densities in the membrane.

Ideally, the ion-step should take place in a negligible time interval with respect to the time constant of the membrane (thus $t_{\text {step }} \ll \tau$ ). Then the steady-state membrane potential as calculated from eqn (5) equals the extremum of the transient $\left(\Delta \phi_{\mathrm{tms}}=\Delta \phi_{\mathrm{m}}\right)$. In this case the TMS equation can be directly used to calculate the extremum of the membrane potentials, without bothering about time-related processes.

\section{The pH-dependent membrane potential}

Under the conditions given in the previous section, the steady-state TMS model may be applied to describe the extremum of the membrane potential $\left(\Delta \phi_{\mathrm{m}}\right)$ as a result of an "ionstep'. The influence of the $\mathrm{pH}$ on $\Delta \phi_{\mathrm{Ims}}$ in the protein/membrane system can now be further investigated.

A protein-loaded membrane will have a $\mathrm{pH}$ dependent charge density, because of the amphoteric nature of the embedded proteins. As long ago as 1977 Kudela et al. indeed showed in a qualitative way that membrane potentials measured with an experimental steady-state system are pH-dependent if amphoteric membranes are used. The membrane potential $\Delta \phi_{\text {Ims }}$ is a function of the effective membrane charge density. In order to describe the membrane potential as a function of $\mathrm{pH}$, during an ion-step experiment, an equation is necessary to relate the charge density to $\mathrm{pH}$. This equation can be derived if the individual dissociation equilibrium constants of all the amino acids in the protein membrane are known. In general, this leads to a dependence of the effective charge density $c_{x}$ on the external $\mathrm{pH}$. However, the individual equilibrium constants of the amino acids are related to their microsurroundings, while moreover the amino acid composition of the protein is often not known. Therefore in a first approximation and based on experimental results of Norde (1986), a simple relationship between $c_{\mathrm{x}}$ and $\mathrm{pH}$ will be used: 


$$
c_{\mathrm{x}}=c_{\mathrm{m}} \sum_{i=0}^{i=n} S_{i} c_{i}\left(\mathrm{pI}_{i}-\mathrm{pH}\right)
$$

where $c_{\mathrm{m}}$ represents a constant with the same dimension as $c_{\mathrm{x}}$. Equation (11) is valid in a region of $\pm 2-3 \mathrm{pH}$ units around the inversion point of the protein-loaded membrane. This charge density versus $\mathrm{pH}$ relation is, in fact, used to derive the linear empirical formula

$$
\Delta \phi_{\mathrm{m}}{ }^{\prime}=\left[\sum_{i=0}^{i=n} S_{i} c_{i}\left(\mathrm{pH}-\mathrm{pI}_{i}\right)\right] \frac{R T}{F} \ln \left(\frac{c_{\mathrm{s} 2}}{c_{\mathrm{s} 1}}\right)
$$

In order to describe $c_{\mathrm{x}}$ over larger $\mathrm{pH}$ intervals, an exponential behaviour of $c_{\mathrm{x}}$ as a function of $\mathrm{pH}$ (saturation) seems more probable. Expression (11) can be empirically adapted to

$$
c_{\mathrm{x}}=c_{\mathrm{m}}\left\{\frac{1-\exp \left[2 \Sigma S_{\mathrm{i}} c_{\mathrm{i}}\left(\mathrm{pI}_{\mathrm{i}}-\mathrm{pH}\right)\right]}{1+\exp \left[2 \Sigma S_{\mathrm{i}} c_{\mathrm{i}}\left(\mathrm{pI}_{\mathrm{i}}-\mathrm{pH}\right)\right]}\right\}
$$

The linear empirical formula (12), can then be rewritten into an exponential empirical equation that accounts for saturation:

$$
\begin{aligned}
\Delta \phi_{\mathrm{m}}{ }^{\prime \prime}= & \left\{\frac{1-\exp \left[2 \Sigma S_{\mathrm{i}} c_{\mathrm{i}}\left(\mathrm{pH}-\mathrm{pI} \mathrm{I}_{\mathrm{i}}\right)\right]}{1+\exp \left[2 \Sigma S_{\mathrm{i}} c_{\mathrm{i}}\left(\mathrm{pH}-\mathrm{pI} \mathrm{I}_{\mathrm{i}}\right)\right]}\right\} \\
& \times \frac{R T}{F} \ln \left(\frac{c_{\mathrm{s} 2}}{c_{\mathrm{s} 1}}\right)
\end{aligned}
$$

This equation reduces to the empirical formula (12), if the exponent is close to zero. It should be noted that the term between braces of eqn (14) has the same structure as the expression for $U$ : $U=\left(1-D^{-} / D^{+}\right) /\left(1+D^{-} / D^{+}\right)$. As will be discussed, the empirical equations seem to have a diffusive behaviour. If diffusion is the main effect, the TMS equation also reduces to the simple diffusion-dependent Nernst-Planck equation: $\Delta \phi_{\mathrm{tms}}=U R T / F \ln \left(c_{\mathrm{s} 2} / c_{\mathrm{s} 1}\right)$. The $U$ versus $c_{\mathrm{x}}$ relation (6), which was derived from the TMS equation, then determines the value of $U$ and therefore the membrane potential.

\section{MATERIALS AND METHODS}

This paper is a theoretical extension of the experimental paper by Schasfoort etal. (1990) published in Biosensors and Bioelectronics. With respect to text length, a brief description of the experiments is given here as a basis for the theory. For a detailed description of the experiments one should consult the paper by Schasfoort et al. (1990).

An ISFET with a lysozyme-loaded membrane deposited on the gate was mounted in a flowthrough system. The $\mathrm{pH}$ of the fluid is measured using a separately placed $\mathrm{pH}$ electrode. By opening a valve for a few seconds the ISFET can be exposed to a higher salt concentration (the socalled ion-step). This resulted in a transient potential detected by the ISFET. The $\mathrm{pH}$ of the electrolyte can be changed by using a gradient vessel. Measuring the transient at different $\mathrm{pH}$ values, the $\mathrm{pH}$-dependent membrane potentials were observed. An important $\mathrm{pH}$ value is the point where the transient is reversed the so-called inversion point $\left(\mathrm{pH}=\mathrm{pI}^{\prime}\right)$. When a high pass filter is used and the $\mathrm{pH}$ electrode is connected to the $X$-input of an $X-Y$ recorder, the transient as a function of $\mathrm{pH}$ is registered. Now, accurate detection of the inversion point ( $\mathrm{pI}^{\prime}$ ) as well as the slope of the $\phi_{\mathrm{m}}-\mathrm{pH}$ curve is possible.

In all experiments it was observed that there is a linear relationship between the membrane potential $\phi_{\mathrm{m}}$ and the $\mathrm{pH}$, with a certain $\mathrm{pl}^{\prime}$ and slope. However, the $\mathrm{pI}^{\prime}$ and the slope of the $\phi_{\mathrm{m}}-\mathrm{pH}$ curve change when the membrane composition is varied in any way. In the $\mathrm{pH}$ region near the inversion point for each value, two $\phi_{\mathrm{m}}$ values are found: the transients are biphasic. The width of this overlap is not always the same, which is explained in the Results and Discussion section. For experimental reasons, the inversion point $\left(\mathrm{pI}^{\prime}\right)$ is defined as the $\mathrm{pH}$ where the positive peak, obtained by an electrolyte step increase is zero (and not the middle point of the overlap region).

The relationship between membrane composition on the one hand and $\mathrm{pI}^{\prime}$ and the slope of the $\phi_{\mathrm{m}}-\mathrm{pH}$ curve on the other proves to be the key feature of the present detection method.

The effect that different concentrations of lysozyme in the membrane have on the shift in inversion point was studied. These experiments also form the basis of the theoretical description of the ion-step-induced response. Lysozyme can be incorporated into the membrane by means of two preparation methods:

(1) Lysozyme can be mixed with polystyrene beads/agarose prior to ISFET coating; a homogeneously loaded membrane is the result. 
(2) Lysozyme can be adsorbed from a solution to a membrane coated ISFET; homogeneity depends on adsorption kinetics.

Only the first procedure has been applied as a basis for the theoretical description.

\section{RESULTS AND DISCUSSION}

In this section, the results obtained from simulations are presented and compared with experimental data obtained from a lysozymeloaded polystyrene beads/agarose membrane system. The intention is to form an opinion on the validity of the equations given in the previous sections. In view of the high isoelectric point of lysozyme $\left(\mathrm{pI}_{1}=11\right)$ and its good adsorbing properties to the polystyrene beads/agarose membrane, this protein was chosen as a model protein. For details concerning the experimental procedures, see also Schasfoort et al. (1990).

\section{$\Delta \phi_{\mathrm{m}}$ as a function of $\mathrm{pH}$}

In the $\mathrm{pH}$ region near the inversion point, two $\Delta \phi_{m}$ values are found for each $\mathrm{pH}$ value; the transients are biphasic. If the standard ion-step procedure is altered (e.g. different $c_{\mathrm{s} 1}, c_{\mathrm{s} 2} / c_{\mathrm{s} 1}$ or another salt is used), it appears that the width of the overlap region is changed. (Some experiments show a width of about $0.5 \mathrm{pH}$ units.) However, a reproducible overlap region is always found for different ImmunoFETs with the same membrane under identical conditions. In addition to the effect of inhomogeneities in the membrane, which was already suggested by Schasfoort et al. (1990) the following effects may contribute to the overlap region.

\section{The effect of ion-association}

If there is a specific interaction of cations and anions with negative and positive fixed charges in the membrane, a screening of the protein charges will be the result. This can be represented by the following equilibria as an example of ionassociation (Moore, 1976; Yamauchi et al., 1987).

$$
\begin{gathered}
\mathrm{COO}^{-}+\mathrm{K}^{+} \stackrel{K_{\mathrm{p}}}{\rightleftarrows} \vdash \mathrm{COOK} \\
\mathrm{NH}_{3}^{+}+\mathrm{Cl}^{-} \stackrel{\kappa_{4}}{\rightleftarrows} \vdash-\mathrm{NH}_{3} \mathrm{Cl} \\
\text { If } K_{\mathrm{p}} \text { differs from } K_{\mathrm{q}}, \text { a different } \mathrm{KCl}
\end{gathered}
$$

concentration results in a different effective charge density of the protein (and also a changed inversion point of the protein membrane). This effect implies that during the ion-step a shift of the inversion point will result as a consequence of the effect of ion-association. An indication that ion-association occurs with the amphoteric charges of proteins is that the titration curve is dependent on the electrolyte concentration that is used (Norde, 1986). Therefore the number of charge units per protein molecule as a function of the $\mathrm{pH}$ is influenced by the type and concentration of the ions present in the sample solution.

\section{The Donnan effect}

If a Donnan potential exists between membrane and solution, a pH difference is the result. The Donnan potential changes as a result of the increased electrolyte concentration, resulting in a different $\mathrm{pH}$ in the membrane. Thus during the ion-step there is a small shift in the membrane charge. However, if an ion-step is carried out with a salt with small intrinsic mobility differences of the ions $(U \approx 0)$, the charge density at the inversion point is very low. Therefore also the Donnan effect resulting in a changed $\mathrm{pH}$ in the membrane will be very small. The $\mathrm{pH}$ change as a result of the Donnan effect of experiments carried out according to the standard procedure is in the range $0.01-0.05 \mathrm{pH}$ units.

In order to fit the experimental $\Delta \phi_{\mathrm{m}}-\mathrm{pH}$ curve with the equations derived in this paper a fit procedure is applied, as shown in Fig. 6. The equations were fitted to the positive experimental values of $\Delta \phi_{\mathrm{m}}$ using as definition for the inversion point $\left(\mathrm{pl}^{\prime}\right)$ the point at which the positive transient is zero. Values of $\mathrm{pI}^{\prime}$ and $\Sigma S_{\mathrm{i}} c_{\mathrm{i}}$ were obtained from the experiment with prescribed $c_{\mathrm{s} 1}$ and $c_{\mathrm{s} 2}$ using the linear empirical equation (eqn (12)) after drawing a best fit through the successive experimental transient maxima.

The extremum of the membrane potential $\left(\Delta \phi_{\mathrm{m}}\right)$ was determined at $\mathrm{pH}=\mathrm{pI}^{\prime}+1$. Subsequently the values of $c_{\mathrm{m}}$ and $c_{\mathrm{s} 2 \text {. ex }}$ were estimated and the values of $c_{\mathrm{x}}$ and $U$ were calculated from eqns (11) and (6) respectively. These values were inserted into the TMS equation (eqn (5)). If $\Delta \phi_{\mathrm{tms}} \neq \Delta \phi_{\mathrm{m}}$, new values of $c_{\mathrm{m}}$ and $c_{\mathrm{s} 2, \text { ex }}$ were estimated. However, if the condition was true, a plot of $\Delta \phi_{\text {tms }}$ versus $\mathrm{pH}$ was calculated with the predetermined values, starting at $\mathrm{pH}=2$ to $\mathrm{pH}=11$. 


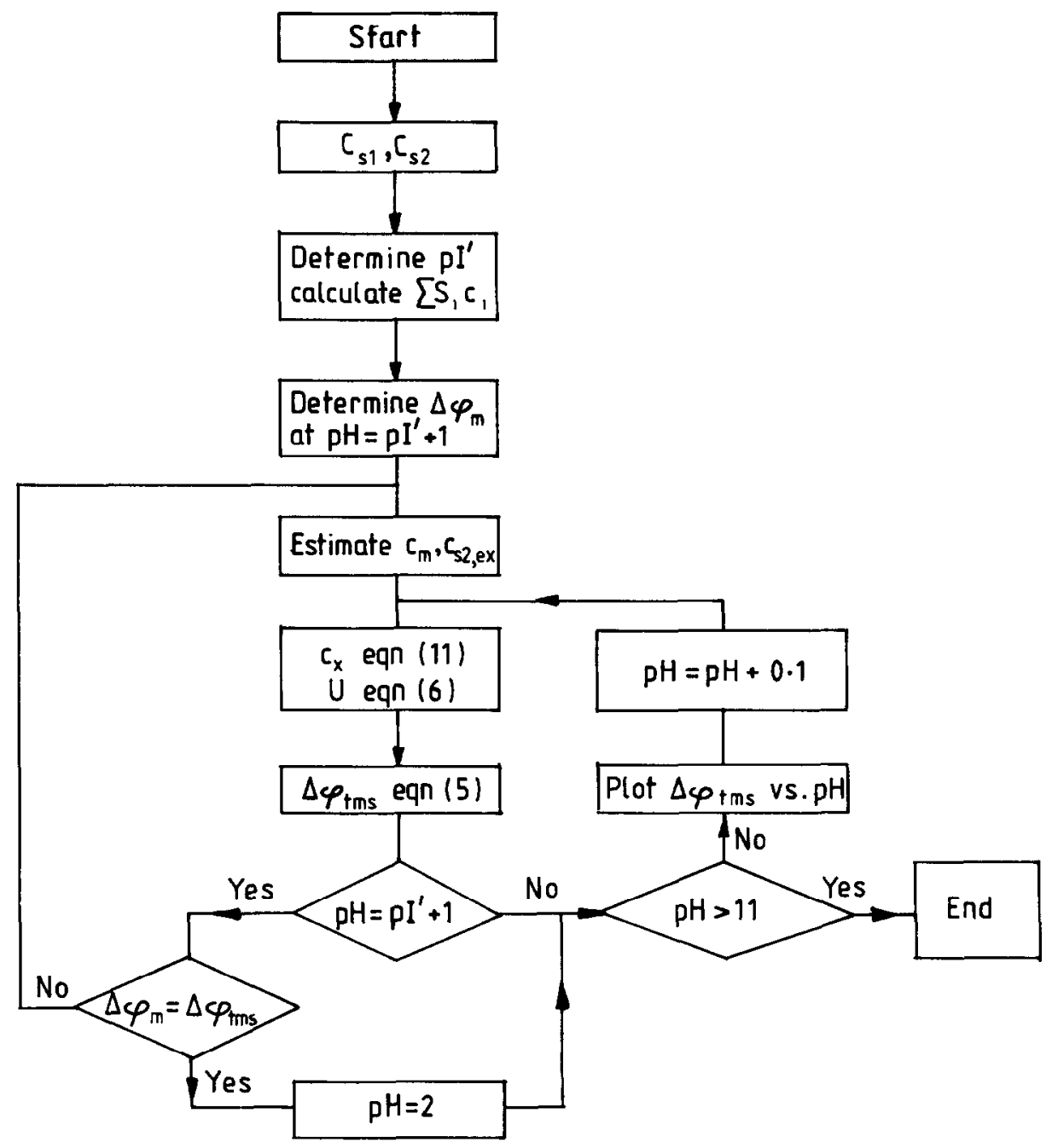

Fig. 6. Outline of the fit procedure (see text).

In Fig. 7 the extremum of the membrane potential as a function of $\mathrm{pH}$ is simulated on the basis of respectively the TMS equation $\left(\Delta \phi_{\mathrm{tms}}\right.$, eqn (5)), the linear empirical formula $\left(\Delta \phi_{\mathfrak{m}}\right.$, eqn (12)) and the exponential empirical formula $\left(\Delta \phi_{m}{ }^{\prime \prime}\right.$, eqn (14)). Also experimental data of the extremum of the membrane potential $\left(\Delta \phi_{m}\right)$ are plotted in the figure.

The following values were used in the simulations: $c_{\mathrm{s} 1}=10 \mathrm{mM}, c_{\mathrm{s} 2}=40 \mathrm{mM}, \Sigma S_{\mathrm{i}} c_{\mathrm{i}}=0.4$, $\mathrm{pI}^{\prime}=4.81, c_{\mathrm{m}}=7.5 \mathrm{mM}, \quad c_{\mathrm{s} 2 \text {. ex }}=3 \mathrm{~mm}$. It is assumed that $t_{\text {step }} \ll \tau$, implying ideal ion-steps.

It is shown in Fig. 7 that at the maxima of the membrane potential, the TMS and the exponential empirical equation approximate the experimental data also at $\mathrm{pH}$ values beyond the linear region. The TMS theory is in satisfactory agreement with the experiments. As already

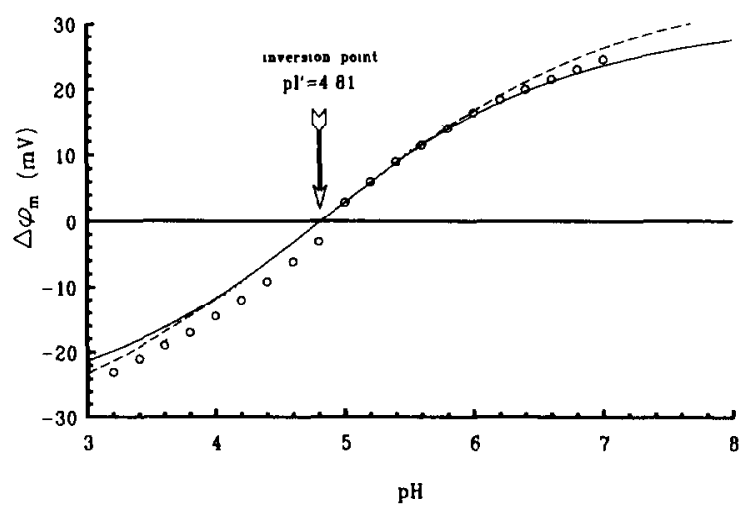

Fig. 7. Ion-step simulations obtained by using the TMS equation. $\left(\Delta \phi_{\text {lms. }}\right)$ the linear empirical equation $\left(\Delta \phi_{m}{ }^{\prime}\right)$ and the exponential empirical equation $\left(\Delta \phi_{m}{ }^{\prime \prime}\right)$ fitted on experimental data $\left(\Delta \phi_{m}\right)$. The parameters used in the equations are: $c_{\sqrt{ }}=10 \mathrm{~mm}, c_{12}=40 \mathrm{~mm}, \quad \Sigma S_{1} c_{1}=0.4$, $p I^{\prime}=4.81 . \mathrm{c}_{m}=7.5 \mathrm{~mm}$ and $\mathrm{c}_{2 .} \mathrm{ex}=3 \mathrm{~mm}$. 
indicated, the overlap region cannot be described by one of the equations.

(Note: it would be reasonable to suggest that the charge density of the membrane is zero at a $\mathrm{pH}$ in the middle of the overlap region, if a salt is used with $U_{0}=0$. But for experimental reasons this method is not followed.)

\section{$\Delta \phi_{\mathrm{m}}$ Under different conditions}

In order to verify the theory with some more experiments, the values of $\Delta \phi_{\mathrm{m}}$ under four different experimental conditions will be discussed. First, the results obtained from different ion-step ratios $\left(c_{\mathrm{s} 2} / c_{\mathrm{s} 1}\right)$ will be shown. Then the $\Delta \phi_{\mathrm{m}}$ versus $\mathrm{pH}$ curves with different initial concentrations $\left(c_{\mathrm{s} 1}\right)$ are presented, followed by ion-step results with different salts. Finally the extremum of the membrane potential was investigated as a function of $\mathrm{pH}$ for different protein concentrations in the membrane.

Case 1: variable ratio $\mathrm{c}_{s 2} / \mathrm{c}_{s 1}$, fixed $\mathrm{c}_{s I}$

Ion-step measurements were carried out at four pH values (and thus charge densities) using a predetermined fixed salt concentration $\left(c_{\mathrm{s} 1}=10 \mathrm{mM} \mathrm{KCl}\right)$ but variable ion-step ratios $\left(c_{\mathrm{s} 2} / c_{\mathrm{s} 1}\right)$. The result of the transient membrane potential as a function of the concentration $c_{\mathrm{s} 2}$ is plotted in Fig. 8 for four different $\mathrm{pH}$ values $\left(\mathrm{pH}=4 \cdot 1,5 \cdot 1,5 \cdot 8\right.$ and $\left.6 \cdot 3, \mathrm{pl}^{\prime}=4 \cdot 81\right)$.

By comparison with Fig. 2 it is concluded from Fig. 8 that all experiments were carried out in the diffusion region where the value of $U$ is the main membrane parameter determining the membrane potential. Further it can be seen that $\Delta \phi_{\mathrm{m}}$ is almost linear in $\log c_{\mathrm{s} 2}$ for each $\mathrm{pH}$, implying that $U$ can be determined from the slope of each curve. If these values of $U$, together with the corresponding values of $c_{\mathrm{x}}$, are inserted into the $U$ versus $c_{\mathrm{x}}$ relation (eqn (6)), it is then found that $c_{\mathrm{s} 2 \text {, ex }}=3 \mathrm{~mm}$ for each of the curves in Fig. 8 . This value is consistent with that obtained from the fit procedure in the previous section.

Case 2: variable $\mathrm{c}_{s l}$, fixed ratio $\mathrm{c}_{\mathrm{s} 2} / \mathrm{c}_{\mathrm{s}}$

In Fig. 9 the experimental values of the transient membrane potential $\Delta \phi_{\mathrm{m}}$ as a function of $\mathrm{pH}$ are plotted for a fixed ratio of $c_{\mathrm{s} 2} / c_{\mathrm{s} 1}=4$, and a variable concentration $c_{\mathrm{s} 1}(5,10$ or $20 \mathrm{mM} \mathrm{KCl})$. The TMS fit for $10 \mathrm{~mm} \mathrm{KCl}$ (the line in Fig. 9) is obtained from the previous section.

It was found that the inversion point shifted to

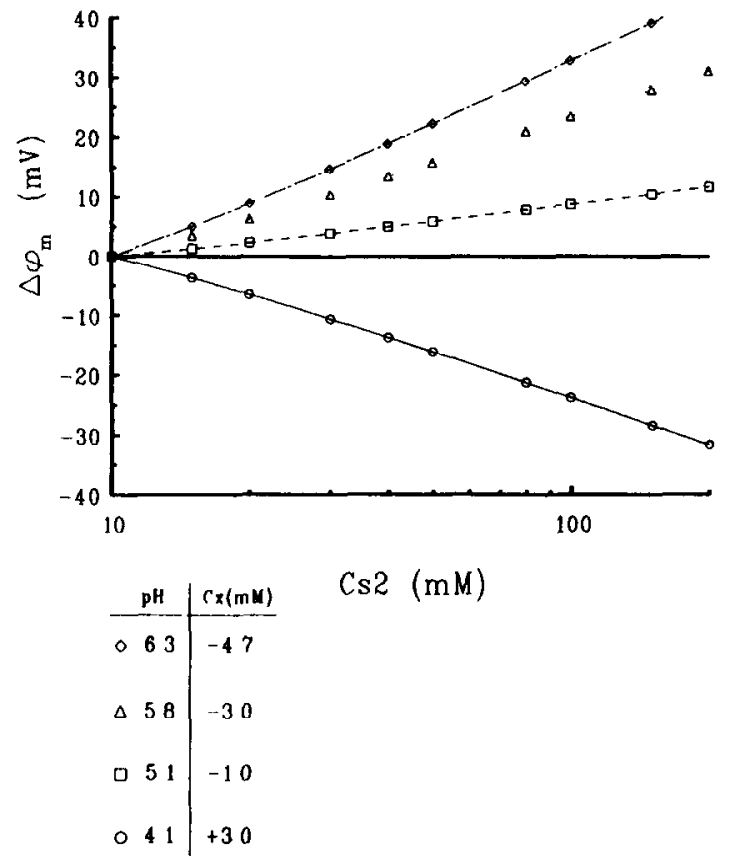

Fig. 8. The extremum of the membrane potential $\left(\Delta \phi_{m}\right)$ as a function of $\mathrm{c}_{\mathrm{s} 2}$ for a fixed salt concentration, $\mathrm{c}_{\mathrm{s} I}=10 \mathrm{mM}$, at four $\mathrm{pH}$ values. The lines represent the result of the TMS simulation, the markers in the figure are from experiments with $\mathrm{pH}$ values of $4 \cdot 1,5 \cdot 1,5 \cdot 8$ and 6.3 respectively. The other parameters for the TMS simulation were the same as used for Fig. 7.

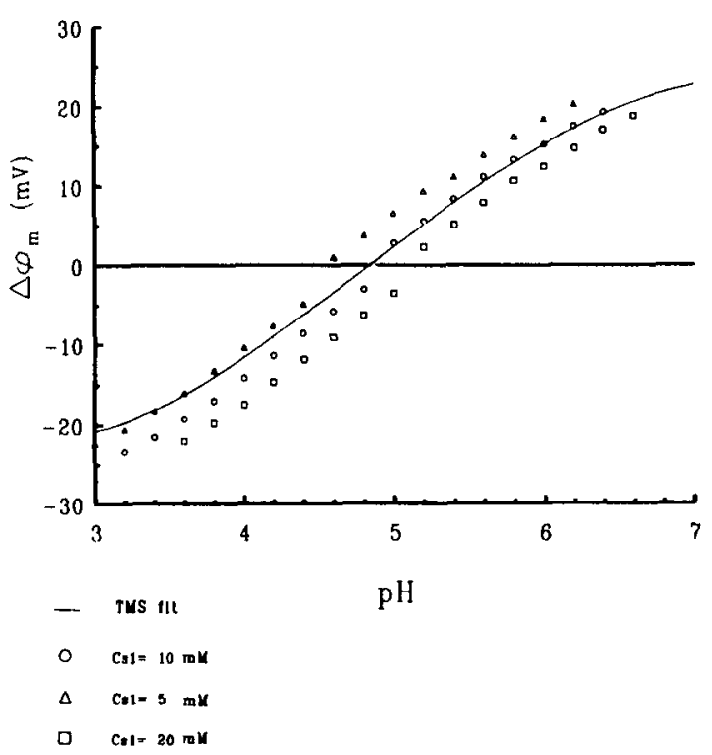

Fig. 9. The extremum of the membrane potential as a function of $\mathrm{pH}$ for a fixed ion-step ratio $\left(\mathrm{c}_{\mathrm{s} 2} / \mathrm{c}_{\mathrm{sl}}=4\right)$ but different initial concentrations $\mathrm{c}_{s l}=5 \mathrm{mM}, \mathrm{c}_{s l}=10 \mathrm{mM}$ and $\mathrm{c}_{s l}=20 \mathrm{~mm}$. Experiments show that the inversion point has changed. The $\mathrm{c}_{s I}=10 \mathrm{~mm}$ curve has been fitted using the values of Fig. 7. 
lower $\mathrm{pH}$ values if lower initial concentrations $c_{\mathrm{s} 1}$ were used $\left(\mathrm{pI}^{\prime}(20 \mathrm{~mm})>\mathrm{pl}^{\prime}(10 \mathrm{~mm})>\mathrm{pI}^{\prime}(5 \mathrm{~mm})\right)$. However, the slope of the $\Delta \phi_{\mathrm{m}}$ versus $\mathrm{pH}$ curve does not change substantially. The shift in the inversion point might be explained by the previously mentioned ion-association. In principle, this effect can be accounted for by an additional term for $\mathrm{pl}^{\prime}$ in eqn (11). However, in view of the speculative nature of this assumption, this method is not followed here. Therefore only one of the experimental curves was fitted, using a pI' corresponding to $c_{\mathrm{s} 1}=10 \mathrm{~mm}$ only.

\section{Case 3: effects of different salts $(\mathrm{KCl}, \mathrm{NaCl}, \mathrm{LiCl})$} In addition to the experiments with $\mathrm{KCl}$, experiments were carried out using $\mathrm{NaCl}$ and $\mathrm{LiCl}$. The results of the experiments are shown in Fig. 10. It was always found that $\mathrm{pI}^{\prime}{ }_{\mathrm{KCl}}>$ $\mathrm{pI}^{\prime}{ }_{\mathrm{NaCl}}>\mathrm{pI}_{\mathrm{LiCl}}^{\prime}$ and that the slopes of the negative part of the $\Delta \phi_{\mathrm{m}}$ versus $\mathrm{pH}$ curves are dependent on the type of cation.

A basic parameter that distinguishes the different salts from each other is the value of $U$ in an uncharged membrane $\left(U_{0}\right)$ (see table in Fig. 10). It is observed that at the inversion point for $\mathrm{KCl}\left(\mathrm{pl}^{\prime}=4 \cdot 81\right)$, the $\mathrm{NaCl}$ and the $\mathrm{LiCl}$ curves exhibit a non-zero membrane potential, which probably has to be ascribed to the intrinsic mobility differences of these cations and anions at zero fixed charge density.

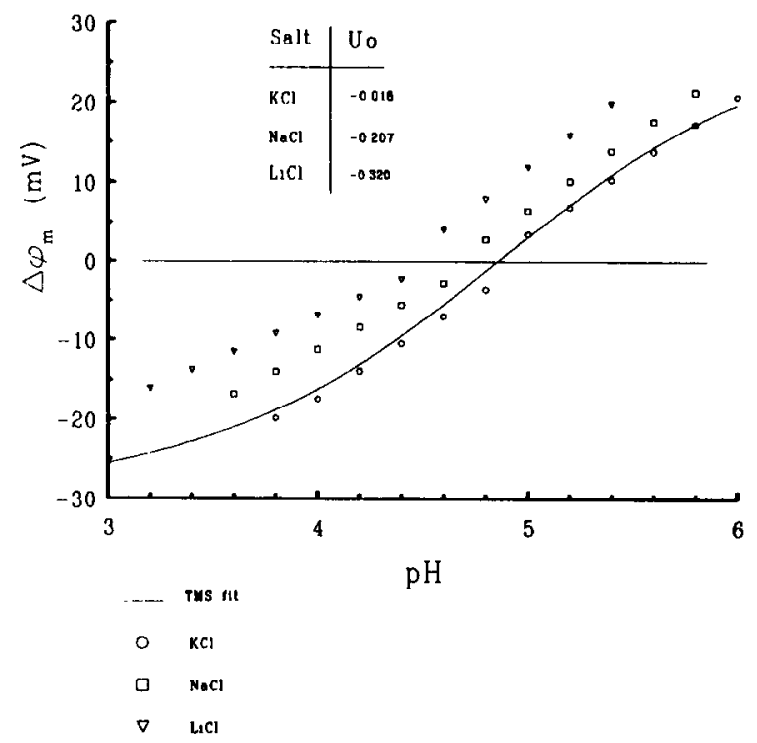

Fig. 10. Ion-step experiments with the salts $\mathrm{KCl}, \mathrm{NaCl}$ and $\mathrm{LiCl}$. The TMS expression was fitted to the $\mathrm{KCl}$ data. Theoretical values of $\mathrm{U}_{0}$ (Moore. 1976) are given in the table.
Furthermore, it is observed that the slopes of the negative parts of the membrane potential versus $\mathrm{pH}$ curves are different from those of the positive ones. This points to the existence of an unknown parameter in the system. A possible explanation of the different slopes might be a different effective maximum charge density at both sides of the inversion point caused by the different degree of ion-association of $\mathrm{NaCl}$ and $\mathrm{LiCl}$ with respect to $\mathrm{KCl}$.

An extra interaction parameter might exist if transport processes take place resulting in an interaction of $\mathrm{Na}^{+}$and $\mathrm{Li}^{+}$with the membrane, which would be different from that of $\mathrm{K}^{+}$. Obviously the different hydration radii of $\mathrm{Na}^{+}$ and $\mathrm{Li}^{+}$with respect to that of $\mathrm{K}^{+}$could contribute to such a parameter. Apparently, the membrane potential is in a certain $\mathrm{pH}$ region mainly determined by that ion of the ion-couple, which has the highest mobility in the membrane. It can be seen from Fig. 10 that at $\Delta \phi_{\mathrm{m}}>0$, where $\mathrm{Cl}^{-}$is the more mobile ion, all curves have approximately the same slope, whereas at $\Delta \phi_{\mathrm{m}}<0$, where the cations have the larger mobility, the slopes of the various curves are dependent on the type of cation. Extension of the experiments to other anions (e.g. $\mathrm{F}^{-}$or $\mathrm{Br}^{-}$) could substantiate these observations.

The TMS curve for the measurement with $\mathrm{KCl}$ as calculated in section $\phi_{\mathrm{m}}$ as a function of $\mathrm{pH}$ is also presented in Fig. 10. For simulation of the $\mathrm{NaCl}$ and $\mathrm{LiCl}$ curves different values of $c_{\mathrm{s} 2 \text {, ex }}$ at different $\mathrm{pH}$ values must be inserted. In this context it would be interesting to repeat the experiments, reported in Case 1 , for the salts $\mathrm{NaCl}$ and $\mathrm{LiCl}$ and to determine $c_{\mathrm{s} 2}$ ex experimentally at different $\mathrm{pH}$ values.

\section{Case 4: different protein concentrations in the membrane}

In Fig. 11 the extremum of the membrane potential as a function of $\mathrm{pH}(\mathrm{KCl}$ ion-step) is given for five different lysozyme concentrations in the membrane $\left(c_{1}\right)$, respectively: $0,6,20,40,80 \mu \mathrm{M}$.

The extrema of the membrane potential as a function of $\mathrm{pH}$ were simulated for different protein concentrations $\left(c_{1}\right)$ using the TMS equation (eqn (5)), $U$ versus $c_{\mathrm{x}}$ equation (eqn (6)) and the $c_{\mathrm{x}}$ versus $\mathrm{pH}$ relationship (eqn (11)), as already described in section $\phi_{m}$ as a function of $\mathrm{pH}$. The following values for the different parameters were inserted in the equations: $c_{\mathrm{s} 1}=10 \mathrm{mM}, \quad c_{\mathrm{s} 2}=40 \mathrm{mM}, \quad c_{\mathrm{s} 2 \mathrm{ex}}=3 \mathrm{mM}$, 


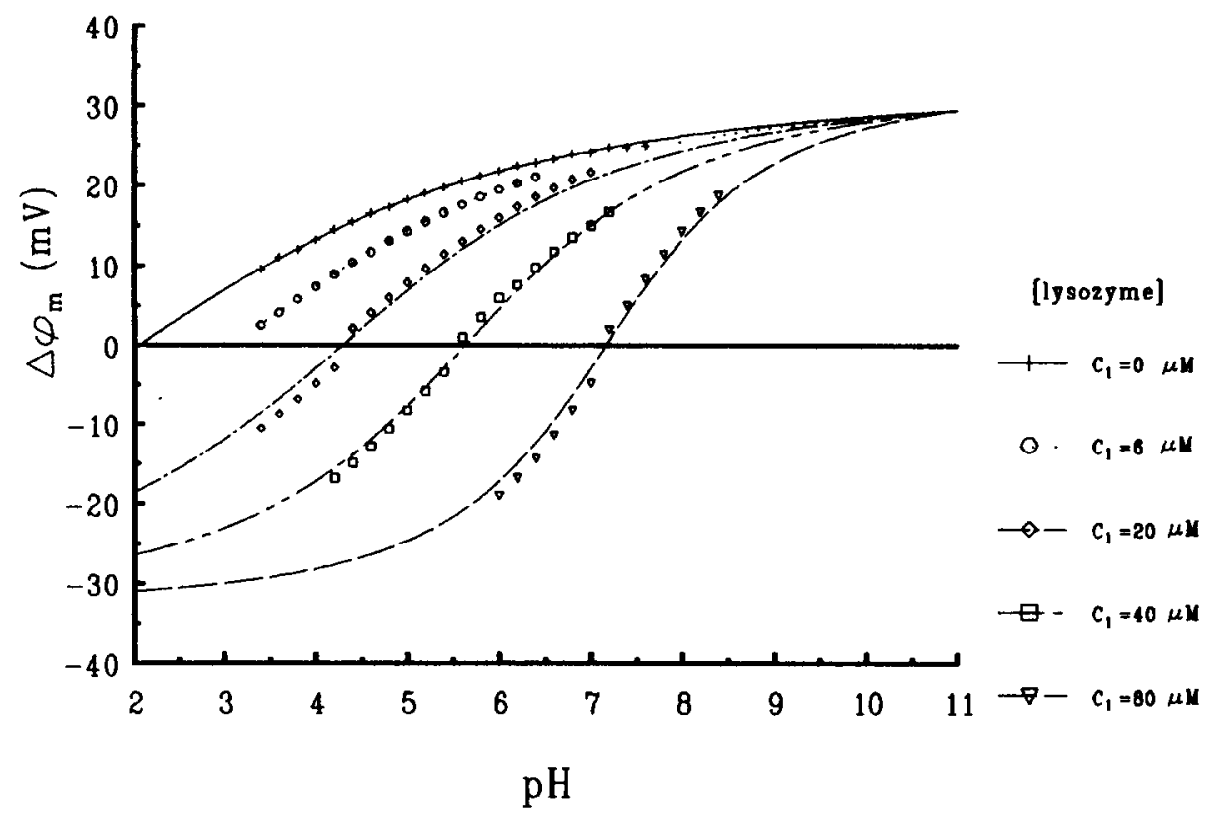

Fig. 11. The extremum of the membrane potential as a function of $\mathrm{pH}$ for different concentrations of lysozyme ( $\mathrm{c}_{1}$ ) in the membrane, namely: $0,6,20,40,80 \mu \mathrm{M}$ respectively. The markers indicate results obtained from experiments. The lines were calculated using the TMS theory with: $\mathrm{c}_{s I}=10 \mathrm{mM}, \mathrm{c}_{s 2}=40 \mathrm{~mm}, \mathrm{c}_{\mathrm{s} 2 \mathrm{ex}}=3 \mathrm{~mm}, \mathrm{c}_{\mathrm{m}}=8 \mathrm{~mm}, \mathrm{~S}_{0} \mathrm{C}_{0}=0.2, p I_{0}=2.05$,

$$
\mathrm{S}_{I}=3 \times 10^{3} \mathrm{M}^{-1} \text { and } p I_{I}=11 \text {. }
$$

$c_{\mathrm{m}}=8 \mathrm{mM}, \quad S_{0} c_{0}=0.2, \quad \mathrm{pI}_{0}=2 \cdot 05, \quad S_{1}=3$ $\times 10^{3} \mathrm{M}^{-1}$ and $\mathrm{pI}_{1}=11$.

\section{CONCLUSIONS}

In this paper the mechanism of potential generation in charged membranes is illustrated and applied to the ion-step detection method. Although the exact influence of the presence of a solid substrate (ISFET instead of an electrolyte solution) on the extremum of the membrane potential is not yet known, the TMS theory could be used satisfactorily in a first approximation to describe the membrane potential for electrolyte/ membrane/solid-state systems. A justification was given for applying a steady-state theory to a nonsteady-state system. It is concluded that only if the ion-step is ideal $\left(t_{\text {step }} \ll \tau\right)$, the extremum of the membrane potential will correspond to the steady-state membrane potential.

In applying an ion-step to an immunoFET, the fixed charge density of the membrane is the response determining parameter, reflected in the extremum of the membrane potential. It was observed that when the ion-step is operated in the diffusion region of the TMS model, the model becomes quite simple and the resulting membrane potential can be predicted easily. In this diffusion region of the model, the effect of the protein-dependent charge density of the membrane on the membrane potential is mainly caused by modulation of the diffusion coefficients. Although the simulations for calculating positive membrane potentials with TMS theory show a satisfactory agreement with all experiments performed with $\mathrm{KCl}$, the whole range of membrane potentials cannot be covered. Neither the TMS equations nor the empirical equations could be applied to fit the experimental data for both positive as well as negative membrane potentials. The reason is that near the inversion point the transients show an unexplained biphasic character, which is probably due to either ion-association effects or $\mathrm{pH}$ changes during the ion-step. A detailed study of ion-steps near the inversion point is necessary to obtain a clear view on this effect.

The experiments with variable initial ion concentrations and fixed ion-step ratios showed that the inversion point is influenced by the initial concentration, which can be explained by assuming ion-association. The fact that ion-steps carried out with $\mathrm{NaCl}$ and $\mathrm{LiCl}$, result in different slopes of the negative part of the $\Delta \phi_{\mathrm{m}}-\mathrm{pH}$ curves, hints at the predominant role that the co-ions play in the generation of $\Delta \phi_{\mathrm{m}}$. The results 
obtained with $\mathrm{NaCl}$ and $\mathrm{LiCl}$ were not fitted to TMS theory, since not all parameters were known. Further verification of the effects, which influence the ion-step induced response, is required to develop a profound theoretical understanding of the results.

\section{ACKNOWLEDGEMENT}

The authors would like to thank Jan Eijkel for his critical reading of the manuscript.

\section{REFERENCES}

Aguilella, V. M., Mafé, S. \& Pellicer, J. (1987). On the nature of the diffusion potential derived from Nernst-Planck flux equations by using the electroneutrality assumption. Electrochim. Acta, 32(3), 483-8.

Aizawa, M. (1987). Immunosensors. Philos. Trans. $\boldsymbol{R}$. Soc. London, 316, 121-34.

Beg, M. N., Siddiqi, F. A., Shyam, R. \& Arshad, M. (1977). Studies with inorganic precipitate membranes. XXV. Evaluation of membrane parameters and test of recently developed fixed charge theories for membrane potential.J. Membrane Sci., 2, 365-74.

Collins, S. \& Janata, J. (1982). A critical evaluation of the mechanism of potential response of antigen polymer membranes to the corresponding antiserum. Anal. Chim. Acta, 136, 93-9.

Demisch, H. U. \& Push, W. (1979). Electric and electrokinetic transport properties of homogeneous weak ion exchange membranes. J. Colloid Interface Sci., 69(2), 247-70.

Demisch, H. U. \& Push, W. (1980). Electrical and electroosmotic transport behaviour of asymmetric cellulose acetate membranes. J. Colloid Interface Sci., 76(2), 445-63.

Higuchi, A. \& Nakagawa, T. (1987). Membrane potential and permeation of salts across bipolar membranes. J. Membrane Sci., 32, 267-80.

Kaibara, K, Nagata, Y., Kimotsuki, T. \& Kimizuka, H. (1986). Study of ion transport across an amphoteric ion exchange membrane - general transport properties of a simple electrolyte. J. Membrane Sci., 29, 37-47.

Kimura. Y., Lim, H. \& Ijima, T. (1984). Membrane potentials of charged cellulosic membranes. $J$. Membrane Sci., 18, 285-96.

Kûdela, V., Vacik, J. \& Kopecek, J. (1977). pH-dependent electrochemical behaviour of hydrophilic ampholytic membranes. Eur. Polym. J., 13, 811-3.

MacGillivray, A. D. (1968). Nernst-Planck equations and the electroneutrality and Donnan equilibrium assumptions. J. Chem. Phys., 48(7), 2903-7.

Meyer, K. H. \& Sievers, J. F. (1936). La permeabilité des membranes. I. Theorie de la permeabilité ionique. Helv. Chim. Acta, 19, 649.

Moore, W. J. (1976). Physical Chemistry. Longman, London.

Morf, W. E., Lindner, E. \& Simon, W. (1975). Theoretical treatment of the dynamic response of ion selective membrane electrodes. Analy. Chem., 47(9), 1596.

Norde. W. (1986). Adsorption of proteins from solution at the solid-liquid interface. Adv. Colloid Interface Sci., 25, 267-340.

Push, W. (1986). Measurement techniques of transport through membranes. Desalination, 59, 105-98.

Push, W. (1976). Membrane potentials of asymmetric cellulose acetate membranes. In Charged Gels and Membranes I. ed. E. Sélégny. Reidel, Dordrecht, pp. 267-76.

Schasfoort, R. B. M., Bergveld, P., Kooyman, R. P. H. \& Greve, J. (1990). A new approach to ImmunoFET operation. Bios. Bioelectron., 5, 103-24.

Teorell, T. (1935). An attempt to formulate a quantitative theory of membrane permeability. Proc. Soc. Exp. Biol. Med., 33, 282.

Yamauchi, A., Okazaki, Y., Kurosaki, R., Hirata, Y. \& Kimizuka, H. (1987). Effect of ionic charge on the electrical properties of an amphoteric ion exchange membrane. J. Membrane Sci., 32, 281-90. 